\title{
Effect of Modified Uvulopalatopharyngoplasty
} without Tonsillectomy on Obstructive Sleep Apnea: Polysomnographic Outcome and Correlation with
Drug-Induced Sleep Endoscopy

This article was published in the following Dove Press journal:

Nature and Science of Sleep

\author{
Feng-Hsiang Chiu (D) ${ }^{1,2}$ \\ Chih-Yu Chen ${ }^{1,2}$ \\ Jih-Chin Lee ${ }^{1,2}$ \\ Ying-Shuo $\mathrm{Hsu}^{3,4}$
}

'Department of Otolaryngology, Head and Neck Surgery, Tri-Service General Hospital, Taipei, Taiwan; ${ }^{2}$ National Defense Medical Center, Taipei City, Taiwan; ${ }^{3}$ Department of Otolaryngology, Shin Kong Wu Ho-Su Memorial Hospital, Taipei, Taiwan; ${ }^{4}$ School of Medicine, Fu Jen Catholic University, New Taipei City, Taiwan
Correspondence: Jih-Chin Lee Department of Otolaryngology, Head and Neck Surgery, Tri-Service General Hospital, National Defense Medical Center, Taipei City, Taiwan

Tel +886-2-87927/92

Fax +886-2-87927I93

Email doc30450@mail.ndmctsgh.edu.tw

Ying-Shuo Hsu

Department of Otolaryngology, Shin

Kong Wu Ho-Su Memorial Hospital,

Taipei City, Taiwan

Tel +886-2-283322II ext.255I

Email sulisky@gmail.com

\begin{abstract}
Objective: Uvulopalatopharyngoplasty (UPPP) is a common procedure for the treatment of obstructive sleep apnea (OSA) and is usually initiated with the resection of palatine tonsils. Because tonsillectomy potentially contributes to complications, minimally invasive upper airway surgeries have been proposed for OSA therapy. Whether tonsillectomy is always essential for UPPP remains unclear, particularly for patients with small tonsils. The purpose of this study was to present the effect of modified UPPP without tonsillectomy (UPsT) on patients with OSA and attempt to select the candidates for this procedure.
\end{abstract}

Methods: This is a retrospective cohort study of patients with OSA, with tonsil size of grade $0-2$, and with only retropalatal obstruction in drug-induced sleep endoscopy (DISE). The patients underwent UPsT at a tertiary center from November 2017 to December 2019. The sleep study was performed before and at least 3 months after surgery. The demographics, surgical outcomes, and staging patterns of preoperative DISE were recorded. The correlation between surgical outcome and DISE was also established.

Results: A total of 22 adults with an average age of 46.5 years [interquartile range: 40 to 60 years] completed the follow-up study. Their apnea-hypopnea index (AHI) and Epworth sleepiness scale values improved significantly after surgery. Of the 17 patients with partial collapse and complete anteroposterior collapse (APC) at the velum, 16 presented good responses to UPsT. However, among the five patients with complete concentric collapse (CCC), only two $(2 / 5,40 \%)$ satisfied the criteria for surgical success. Furthermore, their follow-up AHI values were significantly higher than those of patients without CCC in DISE. Conclusion: UPsT was demonstrated to be an effective therapy for patients with OSA who had small tonsils and retropalatal obstruction in DISE. CCC in sleep endoscopy indicates a poorer surgical outcome than does complete APC and partial collapse at the velum.

Keywords: tonsillectomy, uvulopalatopharyngoplasty, suspension palatoplasty, lateral pharyngoplasty, obstructive sleep apnea, drug-induced sleep endoscopy, complete concentric collapse

\section{Introduction}

Obstructive sleep apnea (OSA) is a prevalent disorder globally that potentially contributes to excessive daytime sleepiness, hypertension, cerebrovascular incidents, type 2 diabetes, and even sudden cardiac death. ${ }^{1-3}$ Continuous positive airway pressure (CPAP) is often used as the first-line treatment, but upper airway 
surgery is considered an alternative therapy because longterm adherence to CPAP treatment was reported to have failed in $25 \%$ to $50 \%$ of the patients with OSA. ${ }^{4}$

Uvulopalatopharyngoplasty (UPPP) is the most common type of sleep surgery for people with snoring, upper airway resistance syndrome, and OSA that are caused by excess tissue in the soft palate and oropharynx..$^{5}$ Review articles have noted that when this procedure is used alone, short-term and long-term success rates range from $35 \%$ to $65 \%$ and from $28.6 \%$ to $55 \%$, respectively. ${ }^{6,7}$ In addition to conventional UPPP, advanced pharyngeal proceduressuch as lateral pharyngoplasty (LP), expansion sphincter pharyngoplasty, barbed reposition pharyngoplasty, and suspension palatoplasty (SP) — have been indicated to increase intraluminal space in the pharynx during sleep. ${ }^{8-12}$ All the aforementioned procedures are initiated with tonsillectomy because the palatine tonsils directly obstruct the pharyngeal space and impede the surgeons' approach to the deep pharyngeal muscles. ${ }^{13}$

Despite the benefits of tonsillectomies, potential complications - such as bleeding, dysphagia, and dysesthesia -are nonnegligible, ${ }^{14}$ particularly for patients with relative surgical contraindications (ie, bleeding tendency). Furthermore, excessive excision of the soft tissue in oropharynx potentially contributed to velopharyngeal insufficiency. ${ }^{15}$ Thus, surgically effective treatments for OSA that minimize invasiveness merit investigation. Various upper airway surgeries without tonsillectomy have been reported in therapies for snoring, upper airway resistance syndrome, and OSA through treatment of the soft palate with tissue trimming, stiffening or reconstruction. ${ }^{16}$ However, the surgical effect on OSA was inevitably concerning because the palatine tonsils and pharyngeal musculature were not treated intraoperatively.

A suture suspension technique was first proposed to pull the palatopharyngeus muscle (PM) laterally and anteriorly, thus advancing the soft palate and expanding the pharyngeal space without tonsillectomy. ${ }^{17} \mathrm{Ma}$ et al reported that this minimally invasive procedure was effective for patients with OSA, with tonsil sizes of $0-2$, and with only retropalatal obstruction during drug-induced sleep endoscopy (DISE). However, systematic reviews have noted insufficient evidence regarding whether tonsillectomy is essential for UPPP treatment in patients with OSA with small tonsils.

In this study, we analyze the surgical outcomes of patients with OSA and small tonsils who underwent modified UPPP without tonsillectomy (UPsT). The correlation between polysomnographic outcome and DISE findings was also investigated. This study clarifies whether tonsillectomy is always necessary for UPPP in patients with OSA, thus aiding sleep surgeons in their selection of candidates for UPsT.

\section{Patients and Methods}

\section{Study Participants}

This retrospective cohort study assessed the responses of 22 patients with OSA to UPsT. The surgeries were all performed by a single surgeon (Dr Chiu, the first author) from November 2017 to December 2019 at a tertiary referral hospital. The inclusion criteria were as follows: (1) The apnea-hypopnea index (AHI) or respiratory event index on baseline attended polysomnogram, or home sleep-apnea test results at $>5$ events/h; (2) BMI $<30 \mathrm{~kg} /$ $\mathrm{m}^{2}$; (3) tonsillar size was classified as grade $0-2$; (4) Friedman tongue position was at stage II or III; ${ }^{18}$ (5) no complete collapse had occurred at the oropharynx, tongue base, epiglottis, or larynx in DISE immediately before upper airway surgery; (6) no prior upper airway surgery had been performed other than nasal surgery; and (7) patients were unwilling to accept CPAP therapy or were nonadherent to CPAP therapy under the care of a boardcertified sleep medicine specialist. The study was approved by the Ethics Institutional Review Board of the Tri-Service General Hospital (TSGHIRB C202005157) and complied with the Declaration of Helsinki. The informed written consent for patients was waived in accordance with the institutional review board's decision given that the participant data being anonymized or maintained with confidentiality in a simple chart and video review.

\section{DISE with Target-Controlled Infusion System}

DISE was performed in a supine position in an operation room immediately before UPsT. Oxymetazoline-soaked pledgets were applied for the vasoconstriction of nasal mucosa before the procedure. Throughout the examination, a target-controlled infusion system (Fresenius Kabi Injectomat TIVA Agilia, Bad Homburg, German) was employed: the system had an initial propofol dose of $1.5 \mu \mathrm{g} / \mathrm{mL}$ that was increased at increments of $0.2 \mu \mathrm{g} / \mathrm{mL}$ to reach anesthesia of absent arousal to loud verbal stimulation and maintain the bispectral index (BIS) level at approximately $60-70 .{ }^{19}$ When the required BIS level was achieved, 
a flexible endoscope was used to assess potential obstructions in the upper airway. The DISE procedure lasted at least 2 minutes long or two to three repeat cycles of snoring, hypoxia, obstruction with apnea and breakthrough with snoring again to ensure reproducible and reliable assessment. All DISE findings were scored by two experienced otolaryngologists on the velum, oropharyngeal lateral walls, tongue base, and epiglottis (VOTE) classification system. ${ }^{20}$

\section{Surgical Procedure and Surgical Success Definition}

All patients with OSA enrolled in the study underwent UPsT. In addition to the preservation of palatine tonsils intraoperatively, the methods of lateral pharyngoplasty $(\mathrm{LP})^{8}$ and suspension palatoplasty (SP) ${ }^{11}$ were combined into the palatopharyngoplasty used in our study. Submucosal fat tissue in the semilunar-shaped supratonsillar area was removed, with careful preservation of the palatopharyngeus muscle (PM). The supratonsillar mucosa was elevated laterally to expose the pterygomandibular raphe, which provided the high tensile strength required to support the suspension sutures of the PM. The PM was partially lysed inferiorly to decrease the vertical tension and thus facilitate the suspension. We used 1-0 Polysorb sutures to secure the PM to the pterygomandibular raphe. Each suture was passed through the pterygomandibular raphe first and then through the PM. After the suspension of PM and wound closure, the soft palate was advanced and thus contributed to the expansion of the retropalatal space. The uvula was either preserved or shortened to $1 \mathrm{~cm}$ in length if required (Figure 1). Finally, we used a 70-degree rigid endoscope to verify the velopharyngeal space through the nose during the procedure in every patient to ensure sufficient velopharyngeal space enlargement.

We defined surgical success using two criteria. The loose criterion was defined as a $\geq 50 \%$ decrease in the AHI relative to the baseline value and a postoperative AHI of $<20$ events $/ h$. The strict criterion was defined as $\mathrm{a} \geq 50 \%$ decrease in the AHI relative to the baseline value and a postoperative AHI of $<10$ events/h.

\section{Statistical Analysis}

Statistical analysis was performed using commercially available software (IBM SPSS statistics 22 for Windows; SPSS, Chicago, IL, USA). Due to the small sample size, we used nonparametric tests in this study; therefore, the quantitative data were reported as median [25th and 752 percentile]. The quantitative data before and after surgery were compared using Wilcoxon signed-rank test. The quantitative post-operative outcomes (ie, post-op

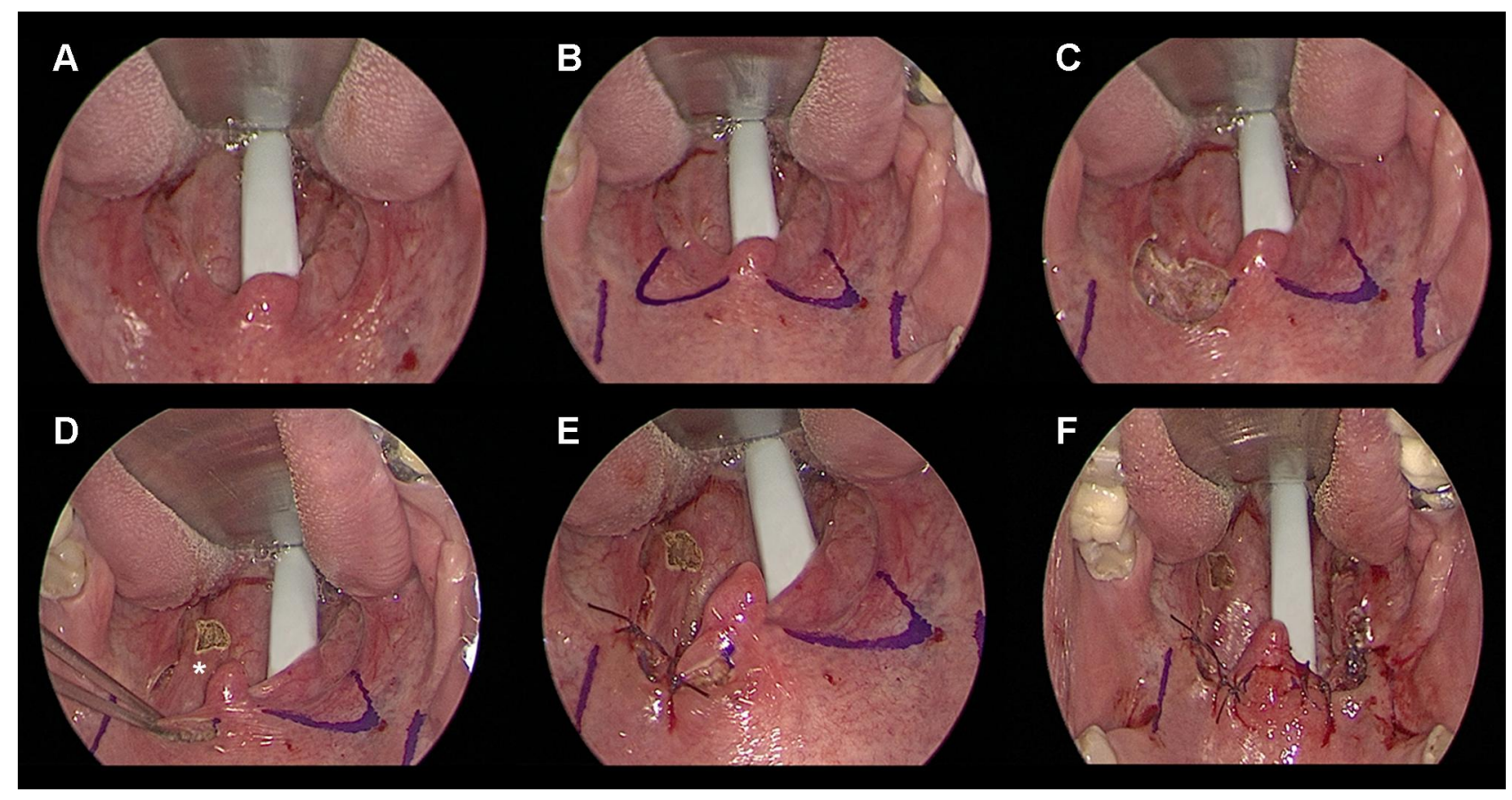

Figure I Surgical view in UPsT. (A) Oropharynx before surgery. (B) Marking of bilateral supratonsillar area and pterygomandibular raphe. (C) Removal of supratonsillar fat with limited muscle destruction. (D) Partial lysis of PM inferiorly; *, PM. (E) Closure of wound to suspend PM and to advance soft palate. (F) Oropharynx after surgery. Abbreviations: UPsT, uvulopalatopharyngoplasty without tonsillectomy; PM, palatopharyngeus muscle. 
AHI) between different preoperative statuses at velum were compared using Mann-Whitney $U$-test with two groups or Kruskal-Wallis test with three groups. The proportion of surgical success between different preoperative statuses at velum were compared using Fisher's exact test. Significance was indicated at a two-sided $P$ value $<0.05$.

\section{Results}

A total of 22 patients who satisfied the inclusion criteria from November 2017 to December 2019 were enrolled; their characteristics are summarized in Table 1 . The upper airway morphology observed in the sleep endoscopy examination of each patient was recorded before surgery. Of the 22 patients, 15 were male and seven were female; they had a median age of 46.5 years [interquartile range (IQR): 40-60 years], a baseline AHI of 22.3 events/h [IQR: $13.1-38.0$ events/ $\mathrm{h}$, and a minimum arterial oxyhemoglobin saturation $\left(\mathrm{SaO}_{2}\right)$ of $83 \%$ [IQR: $79-88 \%$ ]. The follow-up sleep study was conducted at least 3 months after upper airway surgery. The postoperative AHI and Epworth sleepiness scale (ESS) values for all patients were 7.2 events/h [IQR: 3.7-11.7 events/h] and 5.5 [IQR: 3-7], respectively; these values constituted significant improvements from the baseline ( $P<0.001$, Wilcoxon signed-rank test). A total of 18 patients $(18 / 22,81.8 \%)$ and 15 patients $(15 / 22,68.2 \%)$ fulfilled the loose and strict criteria of surgical success after UPsT, respectively. All patients in our study were free of postoperative events except for one patient who presented short-term velopharyngeal insufficiency, which disappeared within 1 week after surgery.

\section{Staging Pattern of Sleep Endoscopy}

At the level of the velum, 15 patients presented with anteroposterior collapse (APC): 13 with complete collapse (Figure 2A and $\mathrm{B}$ ) and the others with partial collapse.

Table I Change in AHI Scores, Oxygen Saturation, and ESS Scores Before and After Surgery

\begin{tabular}{|l|l|l|l|}
\hline Variables & Pre-op & Post-op & $P$ \\
\hline Male/Female, n & $15 / 7$ & - & - \\
Age (years) & $46.5[40.0,60.0]$ & - & - \\
$\mathrm{AHI}$ (events/hours) & $22.3[13.1,38.0]$ & $7.2[3.7,1 \mathrm{I} .7]$ & $<0.00 \mathrm{I}$ \\
Minimum $\mathrm{SaO}_{2}(\%)$ & $83.0[79.0,88.0]$ & $87.0[83.0,89.0]$ & 0.012 \\
ESS total score & $10.5[6.0,14.0]$ & $5.5[3.0,7.0]$ & $<0.00 \mathrm{I}$ \\
ESS $\geq \mathrm{II}$ points, $\mathrm{n}(\%)$ & $\mathrm{II}(50.0)$ & $\mathrm{I}(4.5)$ & 0.002 \\
\hline
\end{tabular}

Note: Data are presented as frequency (percentage) or median [25th, 75th percentile].

Abbreviations: $\mathrm{AHI}$, apnea-hypopnea index; $\mathrm{SaO}_{2}$, arterial oxyhemoglobin saturation; ESS, Epworth sleepiness scale; pre-op, preoperative; post-op, postoperative.
Seven patients presented with concentric collapse: five with complete concentric collapse (CCC, Figure 2C and D) and the others with partial collapse (Table 2). No lateral collapse at the velum was observed in the sleep endoscopy. In addition to collapse at the velum, the sleep endoscopy revealed partial collapse at the tongue base in four patients and at the oropharynx in five patients (data not shown). Patients with complete collapse at the tongue base, oropharynx, epiglottis, and hypopharynx were initially excluded.

\section{Correlation Between Sleep Endoscopy and Surgical Outcome}

Patients were stratified by collapse pattern in the preoperative sleep endoscopy at the level of the velum. The surgical outcomes comprised the follow-up AHI value and the minimum $\mathrm{SaO}_{2}$, AHI reduction ratio, and surgical success rate. The groups of OSA patients with $\mathrm{CCC}$ and other collapse pattern were compared with respect to these parameters (Table 2, Figure 3A).

Noticeably, when patients presented CCC in preoperative DISE with a target-controlled infusion system (TCIDISE), their follow-up AHI values were significantly higher than those of patients with complete APC and partial collapse (Figure 3B). In addition, the surgical success rate (either of loose or strict criteria) was significantly lower in patients with CCC in TCI-DISE. No significant difference was found in the AHI reduction ratio and follow-up minimum $\mathrm{SaO}_{2}$ in patients with preoperative $\mathrm{CCC}$ versus in patients without CCC (Table 2).

\section{Discussion}

Our study analyzed the surgical outcomes for 22 patients with OSA who underwent TCI-DISE and UPsT; 18 patients $(18 / 22,81.8 \%)$ and 15 patients $(15 / 22,68.2 \%)$ fulfilled the loose and strict criteria of surgical success, respectively. Among the 22 patients, significant improvements were observed in AHI, ESS as well as in minimum $\mathrm{SaO}_{2}$ postoperatively. The surgical outcomes of UPsT noted in this study indicated that tonsillectomy is not required for those patients with OSA, with small tonsils, and with only obstruction at the velopharynx in DISE. Moreover, the patients with complete APC and partial collapse of the velopharynx in TCI-DISE presented higher surgical success rates for UPsT compared with patients with CCC. This finding indicates that $\mathrm{CCC}$ at the velum in TCI-DISE is a predictor of poor surgical outcomes from UPsT, as noted by Hsu and Wang. ${ }^{21,22}$ 


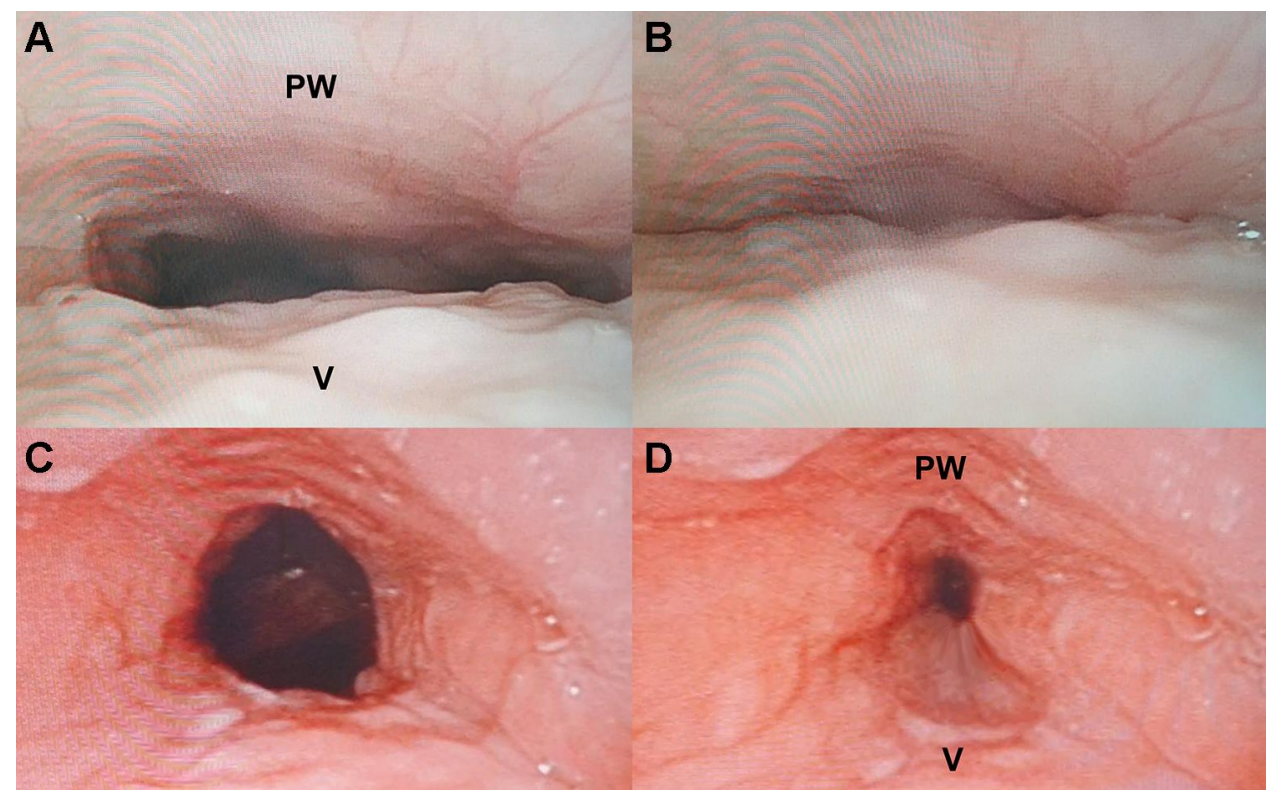

Figure 2 Collapse pattern at velum during TCI-DISE. (A) Expiration view, where complete APC was revealed on inspiration (B). (C) expiration view, where CCC was revealed on inspiration (D).

Abbreviations: APC, anteroposterior collapse; CCC, complete concentric collapse; PW, pharyngeal wall; TCI-DISE, drug-induced sleep endoscopy with target-controlled infusion system; $\mathrm{V}$, velum.

Patients with OSA and with tonsillar size of grade 3 or 4, obesity, or craniofacial anomalies were assumed to be predisposed to airway obstruction during sleep. ${ }^{23}$ According to Friedman, the anatomy of the upper airway determines the surgical success rate of UPPP. Among the patients classified as stage I in Friedman staging (low tongue position and large tonsils), the postUPPP success rate approached $90 \% .{ }^{24}$ However, for patients with OSA and small tonsils, as noted in our study and in the literature, the surgical response to UPPP is significantly decreased. ${ }^{22,24}$ Notably, the patients with OSA with small tonsils enrolled in this study exhibited good responses to UPsT; the surgical success rate was $81.8 \%$ according to the Sher criteria (AHI $<20 / \mathrm{h}$ and $\mathrm{a} \geq 50 \%$ reduction) ${ }^{25}$ This difference in surgical outcomes may have originated from the application of preoperative DISE to exclude those patients with OSA and with complete collapse at the tongue base, oropharynx, and epiglottis. Moreover, combination of the technique of SP and LP into our procedure potentially expanded more retropalatal space than

Table 2 Outcome According to Preoperative Status at Velum

\begin{tabular}{|c|c|c|c|c|c|c|}
\hline $\begin{array}{l}\text { Velum } \\
\text { Status }\end{array}$ & $\mathbf{N}$ & $\begin{array}{l}\text { Post-op AHI } \\
\text { (Events/Hours) }\end{array}$ & $\begin{array}{l}\text { Post-op } \\
\text { Minimum } \mathrm{SaO}_{2}(\%)\end{array}$ & $\begin{array}{l}\text { AHI Reduction } \\
\text { Ratio (\%) }\end{array}$ & $\begin{array}{l}\text { Surgical Success } \\
\text { (Loose Definition)\# }\end{array}$ & $\begin{array}{l}\text { Surgical Success } \\
\text { (Strict Definition)* }\end{array}$ \\
\hline Pre-op velum & & & & & & \\
\hline Non-CCC & 17 & $5.4[2.9,7.8]$ & $88.0[83.0,90.0]$ & $64.9[55.4,78.7]$ & $16(94.1)$ & $14(82.4)$ \\
\hline $\mathrm{CCC}$ & 5 & $22.0[16.1,25.8]$ & $86.0[83.0,87.0]$ & $52.0[33.7,61.8]$ & $2(40.0)$ & I (20.0) \\
\hline$P$ value & & 0.001 & 0.446 & 0.164 & 0.024 & 0.021 \\
\hline Pre-op velum & & & & & & \\
\hline PC & 4 & $1.9[1.5,2.9]$ & $89.5[85.5,92.0]$ & $67.3[59.6,79.4]$ & $4(100)$ & $4(100)$ \\
\hline Total APC & 13 & $7.2[5.2,8.9]$ & $87.0[83.0,89.0]$ & $60.9[55.4,78.7]$ & $12(92.3)$ & $10(76.9)$ \\
\hline $\mathrm{CCC}$ & 5 & $22.0[16.1,25.8]$ & $86.0[83.0,87.0]$ & $52.0[33.7,61.8]$ & $2(40.0)$ & I (20.0) \\
\hline$P$ value & & 0.001 & 0.442 & 0.279 & 0.042 & 0.024 \\
\hline
\end{tabular}

Notes: Data were presented as frequency (percentage) or median [25th, 75 th percentile]; ${ }^{*} \geq 50 \%$ decrease in the $\mathrm{AHI}$ relative to the baseline value and a postoperative $\mathrm{AHI}$ of $<20$ events $/ \mathrm{h} ; * \geq 50 \%$ decrease in the $\mathrm{AHI}$ relative to the baseline value and a postoperative $\mathrm{AHI}$ of $<10$ events $/ \mathrm{h}$.

Abbreviations: AHI, apnea-hypopnea index; CCC, complete circumferential collapse; APC, anterior-posterior collapse; pre-op, preoperative; post-op, postoperative. 

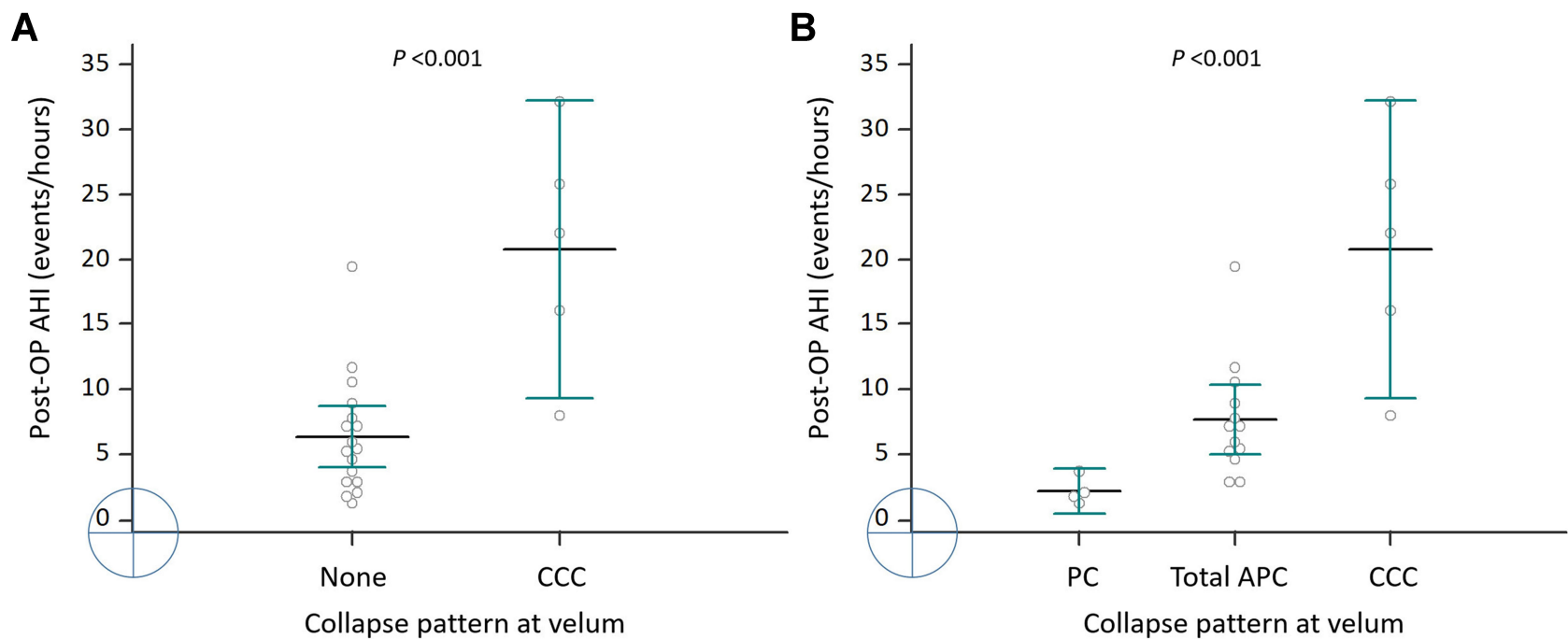

Figure 3 Follow-up AHI values of the 22 patients with dichotomized (A) and detailed (B) CCC pattern.

Abbreviations: AHI, apnea-hypopnea index; CCC, complete concentric collapse; PC, partial collapse; APC, anteroposterior collapse.

conventional UPPP, and thus contributed to such a discrepancy.

DISE is widely considered a useful tool with benefits for decision-making regarding upper airway surgery in patients with OSA. ${ }^{22}$ The correlation between the staging pattern of DISE and surgical outcomes was also investigated. In the literature review, $\mathrm{CCC}$ at the velum was noted to be a potential marker of greater collapsibility of the pharynx ${ }^{26}$ and an independent factor in poor surgical results to palatopharyngoplasty alone. ${ }^{21}$ In addition, CCC was considered to be a contraindication for the advanced technique of upper airway stimulation. ${ }^{27}$ In our study, five patients undergoing UPsT presented CCC in the preoperative sleep endoscopy. All five patients with CCC benefited from the surgery, with a reduction observed in the AHI value, an increase of minimum $\mathrm{SaO}_{2}$ and an improvement in daytime sleepiness (data not shown). However, three patients failed to satisfy the Sher criteria for surgical success. Residual collapse of the upper airway was indicated to be present in the nonresponders postoperatively. Therefore, the presence of CCC in DISE was a poor prognostic factor for UPsT, as described in reports on other surgical procedures. ${ }^{21,22}$ Performing DISE after surgery would be a very interesting way to evaluate the response and correction of collapses. ${ }^{28}$ Nevertheless, postoperative DISE was unavailable in this study for practical and economic reasons; thus, whether a persistent CCC or a newly developed collapse in the upper airway contributed to the poor surgical outcome remains unclear. Notably, most of the other 17 patients without CCC presented good responses to UPsT with significant postoperative improvements in ESS and AHI value. Hence, UPsT was a potentially effective surgery for patients with OSA exhibiting complete APC and partial collapse in preoperative DISE.

In addition to $\mathrm{CCC}$, which was a predictor of poor surgical outcomes, the complete tongue base collapse exhibited during DISE was considered unfavorable to the result of upper airway surgery for patients with OSA. Wang et al described an association between complete collapse at the tongue base and higher rates of nonresponse to UPPP. ${ }^{21}$ Koutsourelakis et al noted complete collapse at the tongue base to be an independent poor prognostic factor for upper airway surgery. ${ }^{29}$ Multilevel upper airway surgery, such as UPPP combined with tongue base reduction, was considered a superior therapy to UPPP alone for the treatment of OSA with complete tongue base collapse. $^{27}$ The patients with complete tongue base collapse were initially excluded from the present study. Therefore, the superiority of UPsT to other procedures with respect to surgical outcomes was attributable to the exclusion of patients with OSA with complete tongue base collapse.

With the evolution of methods for upper airway surgery for OSA, various palatopharyngeal procedures have been proposed for the further expansion of the velopharynx and the oropharynx. ${ }^{8-13}$ In our study, in addition to the resection of unnecessary palatal tissue, the concept of SP was incorporated into UPsT, in which the PM was suspended to advance the soft palate. Anterolateral 
suspension of the PM reportedly benefited the expansion of the retropalatal space more than palatal surgery alone did. ${ }^{11,13}$ We used thick 1-O Polysorb sutures herein to suspend the PM, which potentially facilitated to sustain the expansion of retropalatal space before complete healing of wound. Despite the reposition pharyngoplasty with barbed sutures was effective for the treatment of OSA, ${ }^{10}$ whether conducting barbed sutures in our procedure brings better surgical effect remains unclear. The pterygomandibular raphe, a ligamentous band of the buccopharyngeal fascia that attaches superiorly to the pterygoid hamulus, was essential to providing a reliable structure that supported the suspension sutures of PM in our study and literature review. ${ }^{11,30}$ Furthermore, an LP technique was applied, whereby the PM was partially lysed inferiorly. ${ }^{8}$ This contributed to the decrease in tension between the PM and the pterygomandibular raphe, facilitating the advancement of the soft palate. $^{8}$,

All 22 patients in our study were free of postoperative events except for one patient who exhibited temporary velopharyngeal insufficiency. Moreover, dysphagia after surgery was a concern herein because the PM, which involved in the pharyngeal phase of swallowing ${ }^{31}$ was partially lysed inferiorly. Although destruction of the PM potentially induces swallowing problems, reports of longterm dysphagia after palatopharyngeal surgery were rare in the literature review. ${ }^{8-13,21}$ No patient experienced either short-term or long-term dysphagia in the present study. However, more evidence is required because of this study's small sample size.

Although UPsT resulted in good outcomes in the treatment of patients with OSA with small tonsils, this finding should be interpreted in the context of the following limitations of the present study. First, this report was a retrospective study of few cases (only 22 patients). Despite the significant differences between the groups with respect to PSG parameters, the CCC group was much smaller than the complete APC group. Second, patients with anatomic factors predisposing them to OSA - such as hypertrophic palatine tonsils, morbid obesity, and craniofacial anomalies-were not enrolled. Patients with complete collapse at the tongue base, oropharynx, and hypopharynx were also excluded. The application of UPsT is ordinarily limited to patients with OSA with particular features and not to the whole population. A total of 103 patients experienced preoperative DISE and upper airway surgery within the research period in our institution, but only 22 cases $(21.4 \%)$ met the criteria for UPsT; thus, selection bias is inevitable. Suspension palatoplasty alone or combined with tongue base/hypopharyngeal surgery was preserved for patients with OSA with big tonsils and multilevel collapse in DISE. Third, as noted in other studies, scoring on the DISE is subjective, and reliability is necessarily compromised despite the use of experienced reviewers. Thus, to make our results more consistent, two experienced sleep surgeons reviewed our DISE videos. Upper airway narrowing can be aggravated by sedation and continuous propofol infusion, ${ }^{32}$ contributing to overstaging during DISE. TCI-DISE was used in our study because this technique provides greater accuracy, stability, and safety in the determination of multiple anatomic site obstructions than does the conventional bolus technique. ${ }^{33}$ Finally, the follow-up duration of this study was relatively short (from 6 to 31 months). No PSG data over 1 year after surgery were acquired. Thus, the long-term results of UPsT remain uncertain.

The study's results indicate that UPsT may effectively improve OSA severity and subsequent daytime sleepiness in patients with small tonsils and obstruction at the velopharynx in DISE. Because unilevel palatopharyngoplasty was reported to potentially alleviate multilevel obstruction in the upper airway, ${ }^{21}$ whether UPsT is favorable for patients with OSA exhibiting multiple collapse requires further investigation. Future prospective or larger retrospective studies are required to add clinical significance.

\section{Conclusion}

Our study demonstrated the effect of UPsT on OSA and indicated that tonsillectomy is not always essential for the treatment of OSA, particularly for patients with small tonsils, and with only retropalatal obstruction in DISE. The presentation of CCC in sleep endoscopy indicates a relatively poor surgical outcome, and thus patients with OSA with such DISE findings are not candidates for UPsT.

\section{Highlights}

1. Modified UPPP without tonsillectomy effectively alleviates the severity of OSA.

2. Complete concentric collapse is a poor prognostic factor for modified UPPP without tonsillectomy.

3. Sleep endoscopy facilitates the selection of candidates for modified UPPP without tonsillectomy.

\section{Disclosure}

The authors declare no conflicts of interest for this work. 


\section{References}

1. Engleman HM, Douglas NJ. Sleep 4 : sleepiness, cognitive function, and quality of life in obstructive sleep apnoea/hypopnea syndrome. Thorax. 2004;59(7):618-622. doi:10.1136/thx.2003.015867

2. Tietjens JR, Claman D, Kezirian EJ, et al. Obstructive sleep apnea in cardiovascular disease: a review of the literature and proposed multidisciplinary clinical management strategy. $J$ Am Heart Assoc. 2019;8 (1):e010440. doi:10.1161/JAHA.118.010440

3. Reichmuth KJ, Austin D, Skatrud JB, Young T. Association of sleep apnea and type II diabetes: a population-based study. Am J Respir Crit Care Med. 2005;172(12):1590-1595. doi:10.1164/rccm.200504$637 \mathrm{OC}$

4. Zozula R, Rosen R. Compliance with continuous positive airway pressure therapy: assessing and improving treatment outcomes. Curr Opin Pulm Med. 2001;7(6):391-398. doi:10.1097/00063198200111000-00005

5. Lévy P, J L P, Mayer P, Wuyam B, Veale D. Management of simple snoring, upper airway resistance syndrome, and moderate sleep apnea syndrome. Sleep. 1996;19(9 Suppl):S101-S110. doi:10.1093/sleep/ 19.suppl_9.s101

6. Stuck BA, Ravesloot MJL, Eschenhagen T, de Vet HCW, Sommer JU. Uvulopalatopharyngoplasty with or without tonsillectomy in the treatment of adult obstructive sleep apnea - A systematic review. Sleep Med. 2018;50:152-165. doi:10.1016/j.sleep.2018.05.004

7. He M, Yin G, Zhan S, et al. Long-term efficacy of uvulopalatopharyngoplasty among adult patients with obstructive sleep apnea: a systematic review and meta-analysis. Otolaryngol Head Neck Surg. 2019;161(3):401-411. doi:10.1177/0194599819840356

8. Cahali MB. Lateral pharyngoplasty: a new treatment for obstructive sleep apnea hypopnea syndrome. Laryngoscope. 2003;113 (11):1961-1968. doi:10.1097/00005537-200311000-00020

9. Pang KP, Woodson BT. Expansion sphincter pharyngoplasty: a new technique for the treatment of obstructive sleep apnea. Otolaryngol Head Neck Surg. 2007;137(1):110-114. doi:10.1016/j.otohns.2007.03.014

10. Vicini C, Hendawy E, Campanini A, et al. Barbed reposition pharyngoplasty (BRP) for OSAHS: a feasibility, safety, efficacy and teachability pilot study. "We are on the giant's shoulders". Eur Arch Otorhinolaryngol. 2015;272(10):3065-3070. doi:10.1007/s00405015-3628-3

11. Li HY, Lee LA, Kezirian EJ, Nakayama M. Suspension palatoplasty for obstructive sleep apnea - a preliminary study. Sci Rep. 2018;8 (1):4224. doi:10.1038/s41598-018-22710-1

12. MacKay SG, Carney AS, Woods C, et al. Modified uvulopalatopharyngoplasty and coblation channeling of the tongue for obstructive sleep apnea: a multi-centre Australian trial. J Clin Sleep Med. 2013;9 (2):117-124. doi:10.5664/jcsm.2402

13. Li HY, Lee LA. Relocation pharyngoplasty for obstructive sleep apnea. Laryngoscope. 2009;119(12):2472-2477. doi:10.1002/lary.20634

14. Kezirian EJ, Weaver EM, Yueh B, et al. Incidence of serious complications after uvulopalatopharyngoplasty. Laryngoscope. 2004;114 (3):450-453. doi:10.1097/00005537-200403000-00012

15. Fairbanks DN. Uvulopalatopharyngoplasty complications and avoidance strategies. Otolaryngol Head Neck Surg. 1990;102:239-245. doi:10.1177/019459989010200306

16. Li HY. Palatal surgery for obstructive sleep apnea: from ablation to reconstruction. Sleep Med Clin. 2019;14(1):51-58. doi:10.1016/j. jsmc2018.10.006

17. El-Ahl MA, El-Anwar MW. Expansion Pharyngoplasty by new simple suspension sutures without tonsillectomy. Otolaryngol Head Neck Surg. 2016;155(6):1065-1068. doi:10.1177/0194599816669501
18. Friedman M, Salapatas AM, Bonzelaar LB. Updated Friedman Staging system for obstructive sleep apnea. Adv Otorhinolaryngol. 2017;80:41-48. doi:10.1159/000470859

19. Berry RB, Budhiraja R, Gottlieb DJ, et al. Rules for scoring respiratory events in sleep: update of the 2007 AASM Manual for the Scoring of Sleep and Associated Events. J Clin Sleep Med. 2012;8 (5):597-619. doi:10.5664/jcsm.2172

20. Hohenhorst W, Ravesloot MJL, Kezirian EJ, de Vries N. Druginduced sleep endoscopy in adults with sleep-disordered breathing: technique and the VOTE classification system. Oper Tech Otolaryngol Head Neck Surg. 2012;23(1):11-18. doi:10.1016/j. otot.2011.06.001

21. Hsu YS, Jacobowitz O. Does sleep endoscopy staging pattern correlate with outcome of advanced palatopharyngoplasty for moderate to severe obstructive sleep apnea? J Clin Sleep Med. 2017;13 (10):1137-1144. doi:10.5664/jcsm.6756

22. Wang E, Sun C, Cui X, et al. The role of drug-induced sleep endoscopy: predicting and guiding upper airway surgery for adult OSA patients. Sleep Breath. 2018;22(4):925-931. doi:10.1007/s11325018-1730-7

23. Arens R, Marcus CL. Pathophysiology of upper airway obstruction: a developmental perspective. Sleep. 2004;27(5):997-1019. doi:10.10 93/sleep/27.5.997

24. Friedman M, Vidyasagar R, Bliznikas D, Joseph N. Does severity of obstructive sleep apnea/hypopnea syndrome predict uvulopalatopharyngoplasty outcome? Laryngoscope. 2005;115(12):2109-2113. doi:10.1097/01.MLG.0000181505.11902.F7

25. Sher AE, Schechtman KB, Piccirillo JF. The efficacy of surgical modification of the upper airway in adults with obstructive sleep apnea syndrome. Sleep. 1996;19(2):156-177. doi:10.1093/sleep/ 19.2.156

26. Vroegop AV, Vanderveken OM, Boudewyns AN, et al. Drug-induced sleep endoscopy in sleep-disordered breathing: report on 1249 cases. Laryngoscope. 2014;124(3):797-802. doi:10.1002/lary.24479

27. Liu SYC, Riley RW, Yu MS. Surgical algorithm for obstructive sleep apnea: an update. Clin Exp Otorhinolaryngol. 2020;13(3):215-224. doi:10.21053/ceo.2020.01053

28. Bosco G, Pérez-Martín N, Racionero MA, Plaza G. Expansion sphincter pharyngoplasty: usefulness of DISE. Acta Otorrinolaringol Esp. 2019;70 (4):215-221. doi:10.1016/j.otorri.2018.04.006

29. Koutsourelakis I, Safiruddin F, Ravesloot M, Zakynthinos S, de Vries N. Surgery for obstructive sleep apnea: sleep endoscopy determinants of outcome. Laryngoscope. 2012;122(11):2587-2591. doi:10.1002/lary.23462

30. Lu YT, Tai SK, Lee TL. Pterygomandibular suspension suture: a simple modification of uvulopalatopharyngoplasty for severe obstructive sleep apnea. Eur Arch Otorhinolaryngol. 2018;275 (1):269-273. doi:10.1007/s00405-017-4773-7

31. Sasegbon A, Hamdy S. The anatomy and physiology of normal and abnormal swallowing in oropharyngeal dysphagia. Neurogastroenterol Motil. 2017;29(11):11. doi:10.1111/nmo.13100

32. Hong SD, Dhong HJ, Kim HY, et al. Change of obstruction level during drug-induced sleep endoscopy according to sedation depth in obstructive sleep apnea. Laryngoscope. 2013;123(11):2896-2899. doi: $10.1002 /$ lary. 24045

33. De Vito A, Agnoletti V, Berrettini S, et al. Drug-induced sleep endoscopy: conventional versus target controlled infusion techniques - a randomized controlled study. Eur Arch Otorhinolaryngol. 2011;268(3):457-462. doi:10.1007/s00405-010-1376-y 
Nature and Science of Sleep

Dovepress

\section{Publish your work in this journal}

Nature and Science of Sleep is an international, peer-reviewed, open access journal covering all aspects of sleep science and sleep medicine, including the neurophysiology and functions of sleep, the genetics of sleep, sleep and society, biological rhythms, dreaming, sleep disorders and therapy, and strategies to optimize healthy sleep.

The manuscript management system is completely online and includes a very quick and fair peer-review system, which is all easy to use. Visit http://www.dovepress.com/testimonials.php to read real quotes from published authors.

Submit your manuscript here: https://www.dovepress.com/nature-and-science-of-sleep-journal 